\title{
Determination of the Charge Density Distribution of Polyelectrolytes by Electrophoresis
}

\author{
Kazuki Terajima, ${ }^{*}$ Toru TaKahashi, ${ }^{* *}$ and Mitsuru Nagasawa \\ Department of Synthetic Chemistry, Faculty of Engineering, \\ Nagoya University, Chikusa-ku, Nagoya, Japan.
}

(Received May 7, 1976)

\begin{abstract}
A method of determining the charge density distribution of polyelectrolytes is discussed. Since the electrophoretic mobility of polyelectrolytes is independent of molecular weight, the distribution of charge density of polyelectrolytes can be calculated from the spreading of electrophoretic patterns, provided corrections for the spreading of the boundary due to diffusion and for the polymer concentration dependence of electrophoretic velocity are made. Also the relationship between the electrophoretic mobility and the charge density of the polyelectrolyte needs to be known. Examples of determination of the distribution of the degree of etherification of carboxymethyl cellulose by this method are shown.
KEY WORDS Charge Density Distribution / Electrophoresis / Sodi- um Carboxymethyl Cellulose / Degree of Etherification / Mobility Distribution /

Some polyelectrolytes used for practical purpose, such as, carboxymethyl cellulose, partially hydrolized polyacrylamide, etc., are generally mixtures of the molecules having different charge densities. The physical properties of those polyelectrolytes are affected not only by their average charge densities but also by their charge density distributions. Nevertheless, the effect of charge density distribution on physical properties of polyelectrolytes has not as yet been studied well, partly because the method of determining the distribution of charge density of polyelectrolytes has not been established. This paper is to show that the electrophoretic velocity method is useful for determination of the charge density distribution, i.e., distribution of the degree of etherification (DS) of carboxymethyl cellulose (CMC), which is now widely used in such industrial applications as paste, gums, food additives and in civil engineering works. A few theoretical and pioneering experimental works on this method can be found in the literature, though the theories were given for proteins. ${ }^{1-4}$

* Permanent address: National Chemical Laboratory for Industry, Honmachi, Shibuya-ku, Tokyo.

** Present address: The Aichi Institute for Civil Engineering, Higashi-matabei-cho, Minami-ku, Nagoya.
Various methods may be applicable to determination of the DS distribution of CMC, but, among them, the electrophoretic velocity method appears the most promising because of its reliability and speed. It is now well established from theoretical ${ }^{5-6}$ and experimental ${ }^{7-10}$ studies that the electrophoretic mobilities of linear polyelectrolytes are independent of molecular weight but depend only on charge density provided the molecular weight of the sample and the ionic strength of solution are both high enough.

\section{EXPERIMENTAL}

\section{Samples}

Fifteen samples of CMC, all different in their average DS, were kindly supplied from Dai-ichi Kogyo Seiyaku Co., Ltd. Each sample was dissolved in $1-N \mathrm{NaOH}$ in a nitrogen gas atmosphere and fractionated into three portions at $25^{\circ} \mathrm{C}$ by adding isopropyl alcohol to the solution. The middle fraction was redissolved in water and precipitated with isopropanol, repeatedly, for purification. The sample was precipitated in the form of a powder by adding isopropanol to the solution in the presence of excess $\mathrm{NaOH}$, after the purification had been completed. Then, the excess $\mathrm{NaOH}$ was extracted with isopropanol 
Table I. Characteristics of samples ( $\mathrm{Na} \mathrm{CMC)}$

\begin{tabular}{ccccc}
\hline Sample & $M_{n} \times 10^{-4}$ & $\begin{array}{c}{[\eta],} \\
100 \mathrm{~m} l / \mathrm{g}\end{array}$ & DS & $\begin{array}{c}U, \\
\mathrm{~cm}^{2} / \mathrm{sec} \mathrm{V}\end{array}$ \\
\hline A & 16.5 & 6.19 & $2.0_{1}$ & $3.3_{3}$ \\
B & 13.1 & 5.82 & $1.6_{1}$ & $3.2_{7}$ \\
C & 13.3 & 5.95 & $1.2_{8}$ & $3.1_{7}$ \\
D & $6.5_{2}$ & 3.14 & 0.83 & $2.6_{0}$ \\
E & 14.3 & 8.25 & 0.62 & $2.1_{4}$ \\
(B + C ) & $50: 50$ mixture of B and C \\
(B + D) & $50: 50$ mixture of B and D
\end{tabular}

$[\eta]$ and $U$ are intrinsic viscosity and electrophoretic mobility in $0.1-M \mathrm{NaCl}$ solution at $25^{\circ} \mathrm{C}$, respectively.

containing a slight amount of water. Each purified sample was dehydrated with dry alcohol and ethyl ether and then dried in vacuo.

The DS of samples was calculated from the amount of $\mathrm{Na}_{2} \mathrm{SO}_{4}$ after combustion with sulfuric acid in a platinum dish. The DS values obtained agreed with the values determined by the manufacturer using a different method. The molecular weights of the samples were determined in $0.1-M$ $\mathrm{NaCl}$ solution by a high speed membrane osmometer type 502 of Hewlett Packard. The intrinsic viscosity $[\eta]$ was also determined in $0.1-\mathrm{M} \mathrm{NaCl}$ at $25^{\circ} \mathrm{C}$ by using a capillary viscometer of a modified Ubbelohde type. The molecular characteristics of all five samples thus determined are shown in Table I. The sample No. B, C and $\mathrm{D}$ were employed to test the reliability of the present method for charge density distribution determination.

\section{Determination of Average Electrophoretic Mobility of Samples}

Electrophoresis experiments on CMC were carried out in $0.1-\mathrm{M} \mathrm{NaCl}$ solutions at $25.0_{ \pm}$ $0.03^{\circ} \mathrm{C}$ in a Beckman SPINCO Model $\mathrm{H}$ electrophoresis apparatus with a Rayleigh interference optical system. The cells used were the standard $11 \mathrm{ml}$ cells. The electric current was $5 \mathrm{~mA}$. The method of determination of average electrophoretic mobility of each sample was the same as that reported in previous papers, ${ }^{8-10}$ except that the velocity was calculated from the movement of the central fringe in the Rayleigh interference pattern in the present work, and also that the dialysis of sample solutions against solvent was not carried out in the present experiments. The

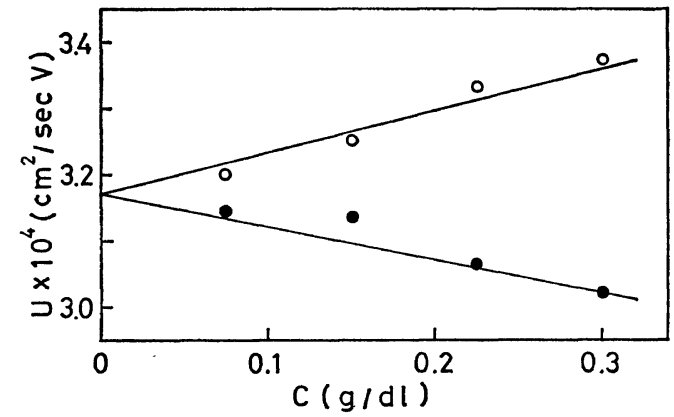

Figure 1. The polymer concentration dependence of average electrophoretic velocity of CMC: Upper, ascending boundary; lower, descending boundary; sample No. C; solvent $0.1-M \mathrm{NaCl}$. $25^{\circ} \mathrm{C}$.

neglect of dialysis is not important if the electrophoretic velocity observed is extrapolated to infinite dilution of polymer and particularly if the ionic strength of the solution is as high as 0.1 . The electrophoretic velocity was determined by plotting the distance of the central fringe from its initial position $(h)$ against time $(t)$ on a graph paper. The plots gave good straight lines as in Figure 1 of ref 10. The electrophoretic velocity at a concentration $u\left(\mathrm{~cm}^{2} / \mathrm{sec} \mathrm{V}\right)$ can be calculated from the slope using the following equation:

$$
u=\left(\frac{\kappa A}{i}\right)\left(\frac{h}{t}\right)
$$

where $A$ is the cross-sectional area of the cell $\left(0.764 \mathrm{~cm}^{2}\right), \kappa$ is the specific conductance of $0.1-M$ $\mathrm{NaCl}$ used as the solvent $\left(1.067 \times 10^{-2} \mathrm{ohm}^{-1} \mathrm{~cm}^{-1}\right)$ and $i$ is the electric current used $\left(5.00 \times 10^{-3} \mathrm{amp}\right)$. The value of $A$ was calculated from the weight of mercury required to fill the cell and $\kappa$ was obtained from a table. To determine the limiting electrophoretic velocity at zero polymer concentration $U, u$ determined at four or five polymer concentrations were extrapolated to zero polymer concentration. An example of the extrapolation is shown in Figure 1. Both curves for ascending and descending boundaries converged at a point, as reported previously, though the scattering of the observed points was larger than in previous works. It was also pointed out that ambiguities arising from skewness of the pattern could be eliminated by this extrapolation. ${ }^{8}$ 


\section{Calculation of Charge Density Distribution from Electrophoretic Pattern}

The electrophoresis experiments for determination of charge density distribution were carried out under the same experimental conditions as above. The electrophoretic mobility is calculated for all points in the integral refractive distribution curves recorded on a photographic plate. The electrophoresis data of potassium poly(styrenesulfonate) (K-PSS) were obtained by the schlieren method in a previous work. ${ }^{10}$ The differential refractive distribution curves recorded were converted to the integral refractive distribution curves by a graphical integration method. The experimental conditions for K-PSS are: Sample, No. F-8 (mol wt $4.85 \times 10^{5}$ ); solvent, $0.1-M \mathrm{KCl}$; polymer concentration, $0.103 \mathrm{~g} / \mathrm{d} l$; temp. $25^{\circ} \mathrm{C}$; current $1.00 \mathrm{~mA}$.

Since the electrophoretic mobility depends only on charge density of polymer if the molecular weight of sample is high enough, the electrophoretic pattern of a sample should show spreading with time if the sample has a distribution of charge density. Therefore, the charge density distribution can be calculated from the boundary spreading if we know the relationship between the electrophoretic mobility and charge density of the polyelectrolyte. However, various corrections may be required to obtain the correct distribution of charge density from electrophoretic patterns. The boundary spreading may be caused not only by the distribution of charge density but also by the concentration dependence of electrophoretic velocity and by diffusion. The problems are similar to the problems in determination of molecular weight distribution of linear polymers by the sedimentation velocity method, which was studied in detail. ${ }^{11}$ The dependence of the boundary spreading on concentration may be eliminated by extrapolating the data at finite concentrations to infinite dilution. The spreading due to diffusion may be eliminated by extrapolating the data at various times to infinite time, i.e., $1 / t=0$, since the boundary spreading due to diffusion is in proportion to $t^{1 / 2}$, whereas the boundary spreading due to DS distribution is in proportion to $t$. The theoretical background for this correction was given by Gosting. ${ }^{11,12}$

Different from the case of sedimentation, moreover, the concentration gradient distributions of a solute at both the ascending and descending boundaries are skewed because of the non-uniform electric field strength at the boundaries even if the solute has a uniform charge distribution. Consequently, the ascending and descending boundaries cannot give an identical integral distribution curve. The error, however, may be eliminated by taking an arithmetic average of the two distribution curves obtained from both boundaries. The reason for the correction method can be easily understood from Figure 1.

Thus, the following method of determining the charge density distribution of a polyelectrolyte is needed: After starting electrophoresis, several photographs are taken at various times, using the Rayleigh interference or schlieren optical system. The apparent integral distribution curve of electrophoretic velocity can be calculated from the difference between the patterns at time 0 and $t$, using eq 1. An arithmetic average $A^{*}(U$, $D, C)$ of the two apparent integral distribution curves calculated from the ascending and descending boundaries is calculated to eliminate the effect of non-uniform electric field strength at the boundaries. Extrapolation of $A^{*}(U, D, C)$ thus determined at various times to $1 / t=0$ gives the integral distribution curve of electrophoretic velocity free from the effect of diffusion $A^{*}(U$, $C)$. It has been shown theoretically that the plot of $u v s .1 / t$ at various points in $A^{*}(U, D, C)$ should be linear at sufficiently large $t .^{11,12}$ The correct integral distribution curve of electrophoretic mobility $A(U)$ can be obtained by extrapolating $A^{*}(U, C)$ at various concentrations to zero polymer concentration. The electrophoretic mobility $U$ can be substituted by DS if we know the relationship between them.

\section{RESULTS}

Potassium poly(styrenesulfonate)s having 100\% degrees of sulfonation have no distribution with respect to charge density. The spreading of their electrophoretic patterns, therefore, is purely due to the diffusion and concentration effects. Figure 2 shows the integral electrophoretic patterns of K-PSS at various times, arithmetically averaged between the curves calculated from the ascending 


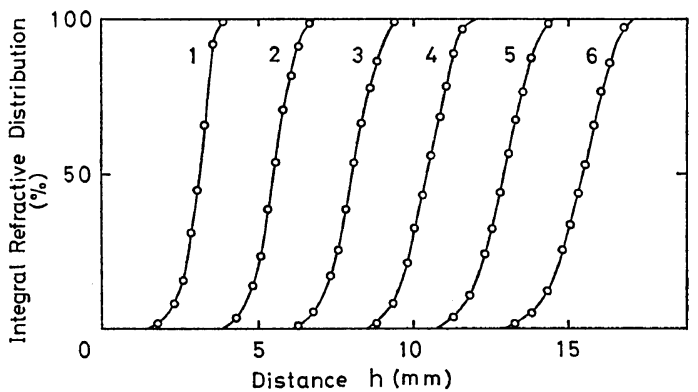

Figure 2. Variation of the integral refractive distribution of potassium poly(styrenesulfonate) (KPSS) with time: sample, K-PSS F-8; mol wt $4.85 \times$ $10^{4}$; polymer conc., $0.103 \mathrm{~g} / \mathrm{d} l$; temp, $25^{\circ} \mathrm{C}$; solvent, 0.1-M KCl solution; current, $1.00 \mathrm{~mA}$; time, (1) 2.5 , (2) 4.5 , (3) 6.5 , (4) 8.5 , (5) 10.5 , and (6) 12.5 hr. The abscissa $h$ denotes the distance from the origin. The data were obtained in a previous work. ${ }^{10}$ The integral distribution curves were converted from the schlieren patterns by graphical integration method.

and descending boundaries. The integral electrophoretic velocity distributions $A^{*}(U, D, C)$ at various times can be calculated from the data in Figure 2, as shown in Figure 3.

To eliminate the spreading of boundary due to diffusion, the electrophoretic velocity $u$ at various points in $A^{*}(U, D, C)$ is plotted against $1 / t$, as shown in Figure 4. As is expected, all graphs tend to a limited range of velocity. The dispersion of $u$ at $1 / t=0$ may be due to the

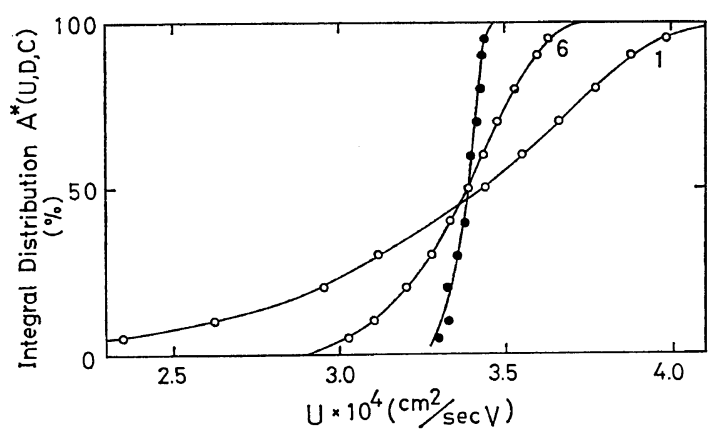

Figure 3. Variation of the apparent integral distribution of electrophoretic velocity $A^{*}(U, D, C)$ of K-PSS in $0.1-M \mathrm{KCl}$. The data are the same as in Figure 2. The experimental data in expt 2-5 are intermediate between in expt 1 and 6 . Filled circles show the values extrapolated to $1 / t=0$ in Figure 4.

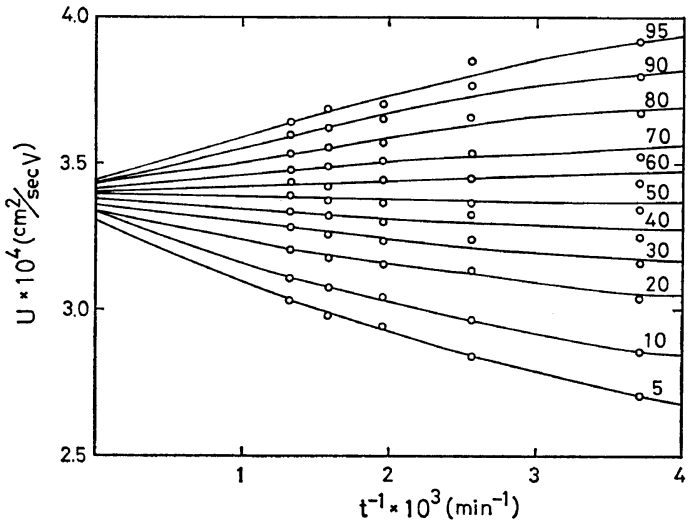

Figure 4. Plot of the electrophoretic velocity $u$ vs. reciprocal time at various points in the apparent integral distribution curve of electrophoretic velocity $A^{*}(U, D, C)$ of K-PSS. The data are the same as in Figure 2. The numbers in the figure show the values $(\%)$ on the ordinate in Figure 3, i.e., $C / C_{0} \times 100$ where $c$ is the concentration at a point in $A^{*}(U, D, C)$ and $C_{0}$ is the total concentration of the sample.

polymer concentration dependence of $u$. The integral distribution of the limiting electrophoretic velocity $u^{\infty}$ obtained by extrapolation, $A^{*}(U, C)$, is also shown in Figure 3 . Figure 3 appears sufficient to conclude that the diffusion effect can be eliminated by this extrapolation method. However, it is observed that the plots of $u v s .1 / t$ in Figure 4 are not linear and, hence, some

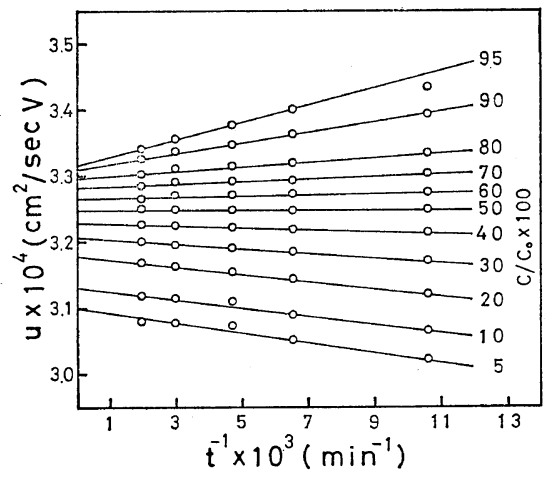

Figure 5. Plot of the electrophoretic velocity $u v s$. reciprocal time at various points in the apparent integral distribution curve of electrophoretic velocity, $A^{*}(U, D, C)$ of CMC: sample CMC, No. C; polymer concn $0.15 \mathrm{~g} / \mathrm{d} l$; solvent, $0.1-M \mathrm{NaCl}$, $25^{\circ} \mathrm{C}$. 
ambiguities may be included in $A^{*}(U, C)$. No further correction for the polymer concentration effect was tried for the data from K-PSS.

The apparent integral electrophoretic velocity distributions of CMC were also determined by the same method. An example of extrapolation of $u v s .1 / t$ plots to $1 / t=0$ for $C M C$ is shown in Figure 5. It is observed that the plots are all linear so that $A^{*}(U, C)$ for these samples can be determined with high reliability. The limiting electrophoretic velocity $u^{\infty}$ at various points in $A^{*}(U, C)$ are plotted against concentration $C$, as shown in Figure 6, to eliminate the polymer concentration dependence of $u^{\infty}$.

The correct electrophoretic mobility distribu-

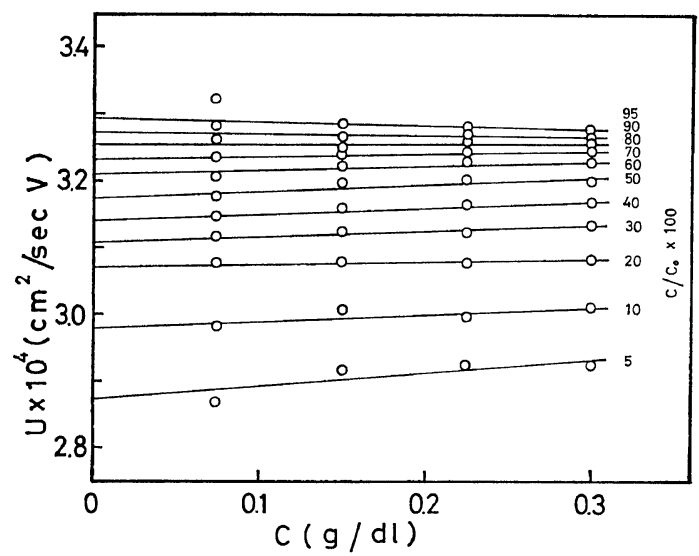

Figure 6. Extrapolation of the limiting electrophoretic velocity at infinite time $u^{\infty}$ to zero polymer concentration at various points in $A^{*}(U, C)$ of CMC: sample $\mathrm{CMC}$, No. C; solvent, $0.1-M \mathrm{NaCl}, 25^{\circ} \mathrm{C}$.

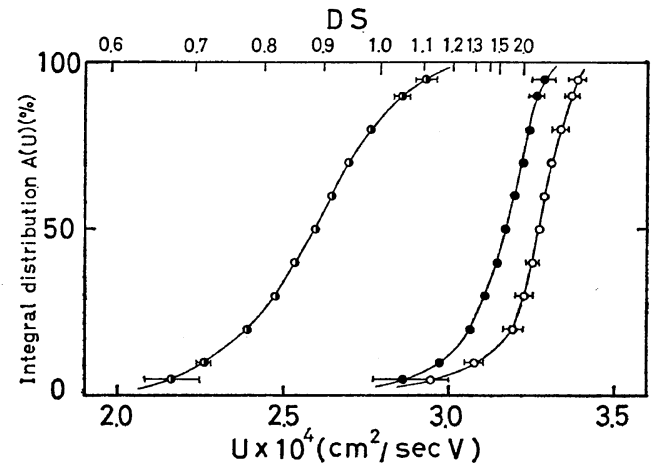

Figure 7. The integral mobility (or DS) distribution curves of CMC sample, B (open), C (filled) and $\mathrm{D}$ (half-filled circles). The standard deviation in the plot of $u^{\infty} v s$. $C$ is shown in the figure.

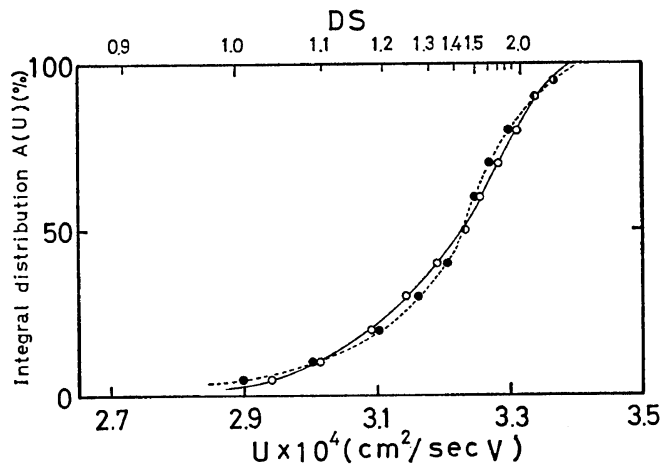

Figure 8. The integral mobility (or DS) distribution of CMC sample, $(B+C)$. Open and filled circles or solid and broken lines denote experimental and calculated values respectively.

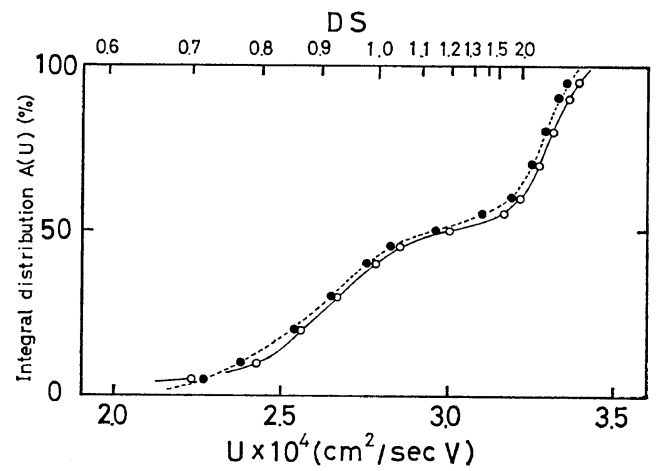

Figure 9. The integral mobility (or DS) distribution of CMC sample, $(\mathrm{B}+\mathrm{D})$. Open and filled circles or solid and broken lines denote experimental and synthetic distribution values, respectively.

tion $A(U)$ of $\mathrm{CMC}$ thus determined for sample No. B, C and D and also for two 50:50 mixtures of $\mathrm{B}$ and $\mathrm{C}$ or D are shown in Figures 7, 8 and 9 , respectively. The measurements for two mixtures were carried out to examine the reliability of this method. The DS of samples No. B and C are close to each other, whereas DS of samples No. B and D are apart. The major part of the experimental error may arise when the data are extrapolated to zero polymer concentration. The standard deviation calculated in the plot of $u^{\infty} v s$. $C$ at various points in $A^{*}(U, C)$ is also shown in those figures.

The synthetic distribution curves $A(U)$ for samples $(B+C)$ and $(B+D)$ calculated from the data for the components (Figure 7) are also shown by dotted lines in Figures 8 and 9, to 


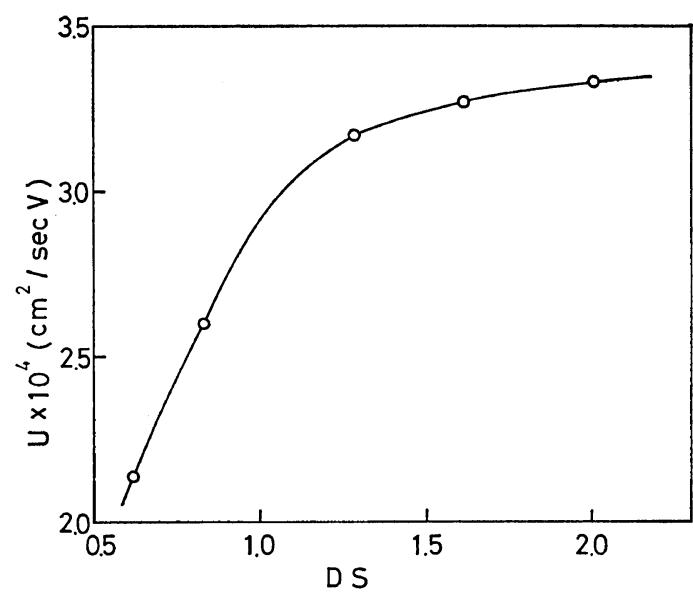

Figure 10. Relationship between the electrophoretic mobility $U$ and the degree of etherification (DS) of CMC. Solvent, $0.1-M \mathrm{NaCl} .25^{\circ} \mathrm{C}$.

be compared with experimental curves. The agreement between the experimental and synthetic curves is satisfactory.

The average electrophoretic mobilities determined for five CMC determined are listed in Table I and also plotted against DS in Figure 10. The electrophoretic mobility in Figures 7-9 were converted to DS by using the curve in Figure 10. The plot in Figure 10 forms a smooth curve despite the fact that those samples have randomly different molecular weights. This is in agreement with a previous conclusion that the electrophoretic mobility of polyelectrolytes is independent of molecular weight. Moreover, Figure 10 shows that $U$ does not significantly increase with DS if DS is beyond about one. This result is also in qualitative agreement with the previous experimental results for partially neutralized polycrylates. ${ }^{9}$ Therfore, the applicability of electrophoretic velocity method to detemination of the charge density distribution of polyelectrolytes may be limited in the range of low charge density.

\section{DISCUSSION}

The linearity between $u$ and $1 / t$ for various points in $A^{*}(U, D, C)$ in Figures 4 and 5 holds at sufficiently long $t$, when the moving boundary in electrophoresis becomes so broad that spreading of the boundary due to diffusion is sufficiently small compared with that due to charge density distribution. Since potassium poly(sty- renesulfonate) has no distribution with respect to charge density, its moving boundary is generally very sharp and, hence, the above limiting law is only valid at very large values of $t$, as can be understood from the data in Figure 4. If the sample has a distribution with respect to charge density, the boundary spreads rapidly so that the limiting linearity between $u$ and $1 / t$ can be easily reached within the period accessible to experiment, as is seen in Figure 5.

Despite the fact that the data in Figure 10 appears to show the presence of an upper limit for the mobility of CMC, the data in Figures 7-9 show that some components have higher mobilities than the limit. This may be caused by the fact that the electrophoretic mobilities in Figure 10 were determined from the velocities of the central fringes in the electrophoresis patterns. Samples having an uniform charge density may be required to determine the relationship between DS and $U$, in order to eliminate this contradiction. Moreover, commercial CMC usually contains low molecular weight fragments, which may have higher mobilities than ordinary molecules having the same DS. Those fragments also can cause an error in experimental results.

Finally, to improve the reproducibility of this method, a somewhat higher concentration of $\mathrm{NaCl}$ may be appropriate for $\mathrm{CMC}$ if we take into account a study on the added-salt effect in sedimentation of $\mathrm{CMC} .^{13}$

Acknowledgment. We wish to thank Dai-ichi Kogyo Co. Ltd. for supplying us the samples and also Mr. N. Kato for his cooperation in measuring the number-average molecular weights of the samples.

\section{REFERENCES}

1. D. G. Sharp, M. H. Hebb, A. R. Taylor, and J. W. Beard, J. Biol. Chem., 142, 217 (1942).

2. R. A. Alberty, J. Amer. Chem. Soc., 70, 1675 (1946).

3. L. J. Bosting, ibid., 74, 1548 (1952).

4. S. Tomita and K. Terajima, Kogyo Kagaku Zasshi (J. Chem. Soc. Japan, Ind. Chem. Sect.), 72, 532 (1969).

5. J. J. Hermans and H. Fujita, Koninkl. Ned. Akad. Wetenschap. Proc. B58, 182 (1955).

6. J. J. Hermans, J. Polym. Sci. 18, 529 (1955). 


\section{Charge Density Distribution of Polyelectrolytes}

7. J. J. Hermans, Chapter 21 in "The Structures of Electrolyte Solutions" John Wiley Press, Inc., New York, N.Y. (1959).

8. M. Nagasawa, A. Soda, and I. Kagawa, $J$. Polymer Sci., 31, 439 (1958).

9. I. Noda, M. Nagasawa, and M. Ōta, J. Amer. Chem. Soc., 86, 5075 (1964).

10. M. Nagasawa, I. Noda, T. Takahashi, and N.
Shimamoto, J. Phys. Chem., 76, 2286 (1972).

11. H. Fujita, "Foundations of Ultracentrifugal Analysis" Wiley-Inter Science, Inc., New York (1975).

12. L. J. Gosting, J. Amer. Chem. Soc., 74, 1548 (1952).

13. S. Tomita and K. Terajima, Nogei Kagaku Kaishi (J. Agr. Chem. Soc. Japan), 39, 144 (1965). 